\section{Anemia de las enfermedades crónicas asociada a obesidad: papel de la hepcidina como mediador central}

\author{
PÍA VILLARROEL H. ${ }^{\mathrm{a}}$, MIGUEL ARREDONDO O. ${ }^{\mathrm{b}}$, MANUEL OLIVARES G.
}

\section{Hepcidin as a central mediator of anemia of chronic diseases associated with obesity}

Recent evidence suggests that obesity-related inflammation may play a central role in hepcidin regulation. Hepcidin is a key regulator of iron homeostasis and has now been suggested as a central mediator of iron metabolism disorders involved in the pathogenesis of anemia of chronic disease. In this review, we focus on subclinical inflammation in obesity and its effect on hepcidin levels, as the most plausible explanation for the relationship between anemia of chronic disease and obesity.

(Rev Med Chile 2013; 141: 887-894). Obesity.

Key words: Anemia, refractory; Antimicrobial cationic peptides; Chronic disease;

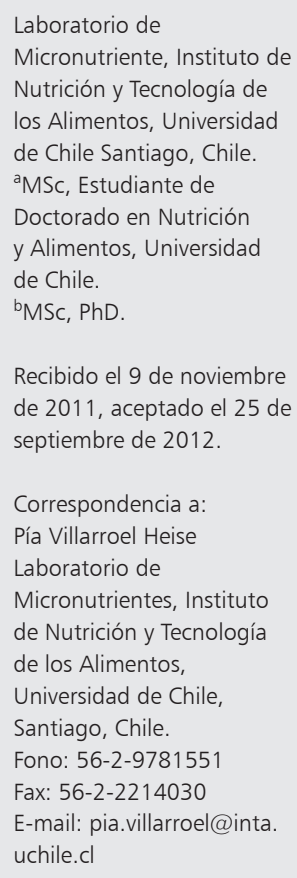

L os desórdenes inflamatorios crónicos desarrollados en el contexto de muchos síndromes infecciosos y enfermedades inflamatorias están asociados con hipoferremia o anemia, conocida bajo el nombre de anemia de las enfermedades crónicas $(\mathrm{AEC})^{1}$. Entre las patologías infamatorias, actualmente la obesidad destaca por su amplia distribución y prevalencia en la población, donde el exceso de grasa corporal está asociado con un aumento de la mortalidad por todas las causas y aumento del riesgo de varias comorbilidades como la $\mathrm{AEC}^{2}$.

Se ha descrito que la obesidad se asocia con bajas concentraciones de hierro $(\mathrm{Fe})$ sérico y con un aumento de la expresión de hepcidina $(\mathrm{Hpc})$ en el tejido adiposo ${ }^{3}$. La elevada producción de Hpc en la obesidad, la convierte en un buen candidato para dar cuenta de la hipoferremia y de la anemia.

La relación inversa entre el nivel de Fe y la adiposidad se informó por primera vez cuando Wenzel y cols. ${ }^{4}$ encontraron una concentración significativamente menor de Fe sérico en pacientes obesos en comparación con los no obesos adolescentes. Recientemente, la masa grasa fue descrita como un factor predictivo negativo de Fe sérico ${ }^{5}$.
La etiología de la AEC asociada a obesidad es incierta y multifactorial e incluye una inadecuada ingesta de Fe en la dieta, mayores requerimientos de Fe de sujetos obesos debido al mayor volumen de sangre, entre otros. Actualmente, se ha sugerido a la Hpc como un regulador clave en los desórdenes del metabolismo de Fe observados en la patogénesis de la AEC, sustentado en el deterioro de la absorción intestinal de Fe, restricción de la liberación de Fe de los depósitos e inadecuada biodisponibilidad de Fe a causa de la inflamación ${ }^{2}$.

\section{Hepcidina}

La hormona $\mathrm{Hpc}$ es un pequeño polipéptido catiónico, rico en cisteínas, de 25 aminoácidos y 4 puentes disúlfuro, miembro de la familia de defensinas que tienen actividad antibacteriana y antifúngica ${ }^{6}$, fue descubierta en el año 2001 por Nicolas et al. ${ }^{7}$, a partir de muestras de orina hu$\mathrm{mana}^{8}$ y plasma humano ultrafiltrado (LEAP-1) ${ }^{9}$. La Hpc es el principal regulador de la absorción de Fe y su distribución en los tejidos y su aumento patológico causa o contribuye al desarrollo de 
$\mathrm{AEC}^{10}$. La expresión del gen que codifica Hpc está casi totalmente restringido al hígado ${ }^{11}$, sin embargo, también se ha determinado su expresión en el tejido adiposo ${ }^{12}$.

La Hpc ha sido considerada como la hormona del $\mathrm{Fe}, \mathrm{y}$ al igual que otras hormonas peptídicas, es sintetizada inicialmente como un péptido más grande, la preprohepcidina de 84 aminoácidos, desde un gen localizado en el brazo largo del cromosoma 19 del genoma humano ${ }^{6}$. La Hpc es procesada a partir del extremo carboxilo terminal en los hepatocitos por la peptidasa señal a prohepcidina ${ }^{13}$. Previo a su secreción, la prohormona convertasa media el procesamiento postraduccional de la $\mathrm{Hpc}$, generando el compuesto bioactivo maduro $^{14}$.

La expresión de Hpc es inducida por las reservas de Fe y la inflamación ${ }^{15}$ y se suprime por la hipoxia y la anemia ${ }^{16}$. Se ha demostrado que el Fe modula la expresión de $\mathrm{Hpc}$ a través de la existencia de vías paralelas que incluyen la activación de las vías de señalización intracelular MAPK/Erk y BMP/SMAD (proteína citoplasmática que media la vía de señalización inducida por BMP $)^{17}$. Se ha postulado que BMP, SMAD y hemojuvelina (HJV) tienen una misma vía de regulación de la expresión de $\mathrm{Hpc}$, ya que la región de respuesta de los 3 estímulos es idéntica ${ }^{18}$. La HJV juega un papel crucial en el mantenimiento del nivel adecuado de la expresión de Hpc y sus efectos están mediados por la mejora de la vía de señalización intracelular BMP/SMAD, mediante el aumento de la sensibilidad de las células a bajos niveles de BMP, actuando como cofactor de activación de receptores de $\mathrm{BMPs}^{19}$.

Por otro lado, las citoquinas proinflamatorias desempeñan un rol primordial en la inducción del gen de la Hpc durante la inflamación, produciendo un rápido aumento de la $\mathrm{Hpc}$ urinaria, con una disminución concomitante en los niveles de Fe sérico, por lo que se le considera como una proteína de fase aguda tipo $\mathrm{II}^{20}$. La potencial actividad proinflamatoria de interleuquina-6 (IL-6) es un fuerte estímulo para la producción de Hpc mediante la activación de la vía de señalización intracelular JAK/STAT ${ }^{21}$. IL-6 interviene en la inducción de la síntesis de Hpc por el aumento de la unión del factor de transcripción STAT3 al promotor de la $\mathrm{Hpc}^{22}$, por lo que se ha descrito que IL-6 parece jugar un papel clave en el aumento de la expresión de Hpc en respuesta a la inflamación.
La actividad de Hpc comienza con la unión a la cara extracelular de su receptor y único exportador celular de Fe conocido, la ferroportina (Fpn $)^{23}$. La Fpn es una proteína transmembrana que se expresa en tejidos que transportan grandes cantidades de Fe: 1) enterocitos duodenales que absorben Fe desde la dieta; 2) macrófagos del hígado, bazo y médula ósea que reciclan el Fe de los eritrocitos senescentes; 3) hepatocitos que almacenan y liberan el Fe de acuerdo a las necesidades del cuerpo y 4) trofoblasto placentario que transporta el Fe de la madre a la circulación fetal ${ }^{24}$.

La unión de la Hpc a Fpn en las membranas de células exportadoras de Fe induce la internalización del complejo receptor-ligando, con una posterior ubiquitinización del péptido y degradación en el lisosoma ${ }^{25}$. La pérdida de la Fpn causa retención de $\mathrm{Fe}$ en los macrófagos, disminución de la absorción de Fe dietario a nivel del enterocito y una posterior disminución del flujo de Fe hacia el plasma ${ }^{26}$, por lo que es considerado como un regulador negativo de la absorción de Fe, regulando su absorción en respuesta a los requerimientos corporales del mineral.

Por lo tanto, y a partir de los datos proporcionados, se ha demostrado que la síntesis de Hpc se correlaciona inversamente con la expresión de Fpn duodenal y que esto afecta la absorción de $\mathrm{Fe}^{27}$, convirtiendo al sistema Hpc- Fpn en el blanco terapéutico en las anemias y desórdenes por sobrecarga de Fe.

\section{Obesidad y hepcidina}

La Hpc es producida principalmente por el hígado, pero se ha reportado una mayor expresión en el tejido adiposo de obesos, lo que demuestra una mayor asociación entre Fe plasmático y adiposidad en niños y adolescentes ${ }^{28}$, hombres y mujeres adultos ${ }^{29} \mathrm{y}$ mujeres postmenopáusicas ${ }^{30}$.

El tejido adiposo es un órgano endocrino activo, secretor de numerosas hormonas y citoquinas proinflamatorias que contribuyen al desarrollo de la inflamación asociada ${ }^{31}$. En pacientes obesos la infiltración de macrófagos ${ }^{32}$ induce la producción de una serie de adipoquinas que están vinculadas a la respuesta inflamatoria, incluyendo, factor de necrosis tumoral- $\alpha$ (TNF- $\alpha$ ), IL-1 $\beta$, IL-6, IL-8 e IL- $10^{33}$. Este antecedente parece explicar el rol de la Hpc como modulador entre la inflamación de la obesidad y la AEC. 
Adicionalmente, el tejido adiposo, especialmente visceral, provoca hipoxia adipocitaria, que resulta en una inflamación crónica producto de las diferencias en la tensión de oxígeno que producen una marcada diferencia en la expresión de citoquinas inflamatorias que finalmente pueden aumentar la expresión de Hpc y desempeñar un papel en la asociación entre la obesidad y el bajo nivel de $\mathrm{Fe}^{34}$.

Sumado a estos aspectos, el tejido adiposo puede influir en la homeostasis del Fe en pacientes obesos mediante la regulación de la vía de señalización $\mathrm{BMP} / \mathrm{HJV}$. Se ha descrito la expresión de HJV en el tejido adiposo, lo que podría estar involucrado en la regulación de la expresión de Hpc en este tejido ${ }^{35}$.

La relación entre obesidad e inflamación es apoyada por la presencia de obesidad con elevados niveles plasmáticos de muchas proteínas de fase aguda, como ferritina sérica ${ }^{36}$ y proteína $\mathrm{C}$ reactiva $^{37}$. La síntesis de ferritina, está regulada por IL-6 y TNF- $\alpha$, insulina, factor de crecimiento insulínico (IGF-1) y hormonas tiroideas ${ }^{38}$. Por lo tanto, la obesidad está asociada a un aumento de las reservas de $\mathrm{Fe}^{39}$, implicando una acumulación excesiva de $\mathrm{Fe}$ en los tejidos del cuerpo, especialmente en el hígado, que promueven la generación de especies reactivas del oxígeno y daño tisular².

En relación a esto, se ha propuesto un síndrome de sobrecarga de Fe en pacientes con incremento de ferritina sérica y saturación de transferrina normal, donde la gran mayoría de estos pacientes tiene sobrepeso, hiperlipidemia, hipertensión arterial o un metabolismo anormal de la glucosa. Se ha observado que los niveles de ferritina sérica se correlacionan positivamente con la glucosa e insulina sérica en ayunas y negativamente con la sensibilidad a la insulina ${ }^{2}$ y en consecuencia, hay evidencia de que la hiperferritinemia observada en la obesidad debe ser interpretada en el contexto del síndrome de resistencia a la insulina (RI).

La inflamación crónica de bajo grado, característico de la obesidad, puede contribuir al desarrollo de RI, intolerancia a la glucosa y diabetes mellitus tipo $2(\mathrm{DM} 2)^{40}$. La insulina provoca una estimulación rápida de la absorción de Fe en adipocitos y hepatocitos ${ }^{41} \mathrm{y}$ el Fe interfiere con la acción de la insulina en el hígado, contribuyendo a la RI a través de mecanismos relacionados tanto con la capacidad de sensibilización como la secreción de insulina ${ }^{42}$. Además, la insulina posiblemente activa el factor inducible por hipoxia $1 \alpha$ (HIF$1 \alpha$ ), que potenciaría los efectos inflamatorios del tejido adiposo ${ }^{43}$.

La acumulación de tejido adiposo intraabdominal es, al menos en parte, responsable del desarrollo del síndrome metabólico, ya que el exceso de grasa abdominal está involucrado en la fisiopatología de la RI, mientras que su evaluación clínica, la circunferencia de cintura, es uno de los criterios que definen el síndrome metabólico ${ }^{44}$. En relación a esto, existe una asociación positiva entre el síndrome metabólico y niveles elevados de ferritina sérica ${ }^{45}$.

Entre las adipoquinas secretadas por el tejido adiposo, la leptina y la adiponectina cobran gran importancia desde el punto de vista metabólico. La leptina, adipoquina proinflamatoria ${ }^{46}$, puede operar por un mecanismo de señalización intracelular similar a la IL- $6{ }^{47}$. Estudios in vitro reportaron que la leptina regularía la expresión hepática de Hpc, sugiriendo que el aumento de esta adipoquina en sangre podría contribuir a los desórdenes del metabolismo de $\mathrm{Fe}^{48}$. En niños obesos se ha mostrado que el aumento de la producción de Hpc está mediado por la leptina ${ }^{49}$.

Por otro lado, la adiponectina es una adipoquina antiinflamatoria con efectos metabólicos contrapuestos a la leptina ${ }^{50}$. Estudios recientes han demostrado que el tejido adiposo de ratones ob/ob, que presentan una mutación en el gen de la leptina, es más hipóxico en relación al tejido adiposo de ratones delgados, observando además una asociación entre hipoxia e incremento en la expresión de genes inflamatorios y disminución de la expresión de la adiponectina en el tejido adiposo $^{51}$ (Figura 1).

\section{Hepcidina y anemia de las enfermedades crónicas}

La AEC, también llamada "anemia de la inflamación", es la segunda de las anemias más frecuente después de la anemia causada por deficiencia de Fe (ADF) y en la población de pacientes hospitalizados es la anemia que se presenta con mayor prevalencia ${ }^{52}$. La AEC fue descrita hace más de 50 años como anemia normocítica, normocrómica, pero puede ser microcítica e hipocrómica conforme avanza la enfermedad ${ }^{1}$, que se caracteriza bioquímicamente por bajo Fe plasmático, 
disminución de la capacidad de unión de Fe total, disminución de la saturación de transferrina (Tf), disminución de sideroblastos, aumento del Fe retículo endotelial y valores normales de receptor de transferrina sérico (RTf) ${ }^{53,54}$.

En la práctica clínica, es importante y difícil diferenciar la AEC de la ADF debido a que ambas patologías se presentan con disminución de la concentración de Fe sérico y de la saturación de Tf. Además, durante la inflamación, se dificulta la interpretación de los niveles de ferritina debido a que su expresión es inducida por la sobrecarga de Fe y por las citoquinas inflamatorias. Para estos efectos, es importante contar con información sobre las concentraciones circulantes de Hpc en el plasma, medición que se dificulta debido a la falta de un método ampliamente disponible. Actualmente, se reconoce a la espectroscopia de masas y el método de ELISA (enzyme-linked immunosorbent assay) como técnicas promisorias para la determinación de $\mathrm{Hpc}$, lo que puede ayudar en la selección de una terapia apropiada para estos pacientes ${ }^{55}$.

Se ha propuesto que la relación $\mathrm{RTf} /$ logaritmo ferritina sérica sería de utilidad para evaluar la nutrición de $\mathrm{Fe}$ en sujetos con inflamación crónica, es así, que una relación $<1$ sería indicador de AEC y si es $>2$ indicaría depósitos insuficientes de Fe, independientemente si hubiera o no una $\mathrm{AEC}^{54,56}$. El RTf es la vía convencional por el cual las células adquieren Fe para los requerimientos fisiológicos, bajo condiciones de deficiencia de Fe hay una mayor concentración de RTf en la superficie celular, como un mecanismo para secuestrar el Fe que se necesita ${ }^{54}$ y su medición ha sido aceptada como el índice óptimo para definir la deficiencia de Fe tisular ${ }^{57}$. Por otro lado, la ferritina sérica sigue siendo el mejor indicador de las reservas corporales de $\mathrm{Fe}^{58}$.

La primera evidencia directa que apoya la función de la $\mathrm{Hpc}$ en la AEC viene de pacientes con una enfermedad de los depósitos de glucógeno (GSD) tipo 1a. La GSDla es un trastorno autosómico recesivo causado por mutaciones en el gen de la glucosa-6-fosfatasa, resultando en una incapacidad para mantener la homeostasis de glucosa. Pacientes adultos con GSD1a desarrollaron grandes adenomas hepáticos y anemia con rasgos característicos a la $\mathrm{AEC}^{59}$.

Niveles elevados de Hpc se han asociado con el desarrollo de AEC, patología caracterizada por una disminución de la movilización y biodispo- nibilidad de $\mathrm{Fe}$, que da origen a una hipoferremia y a un aumento de las reservas de Fe celular ${ }^{60}$. En pacientes que presentan AEC se han detectado elevadas concentraciones de $\mathrm{Hpc}$, debido a trastornos inflamatorios o infecciosos que han aumentado notablemente la excreción urinaria de $\mathrm{Hpc}^{11}$.

La hipoferremia resultante es presumiblemente un componente de la respuesta inmune innata, observándose que el aumento de la expresión de esta proteína en respuesta al estímulo inflamatorio puede servir como estrategia defensiva del hospedero, que secuestra el Fe de los microorganismos infecciosos, impidiendo el acceso de los agentes bacterianos infecciosos al Fe, esencial para su crecimiento y multiplicación ${ }^{61}$, sin embargo, también conduce a deficiencia en la disponibilidad de Fe para la eritropoyesis, ya que no puede ser eficientemente movilizado desde los lugares de depósito hacia los precursores eritroides. En la AEC, el Fe está presente en el cuerpo pero mal distribuido, siendo secuestrado en los macrófagos, y por tanto, encontrándose deficiente para la eritropoyesis ${ }^{62}$.

La patogenia de la AEC es un fenómeno complejo en el que existe una inhibición de la eritropoyesis por diferentes mecanismos; una activación directa de algunas citoquinas que median la respuesta inflamatoria e inmune, ocasionando un deterioro en la producción de eritropoyetina $(\mathrm{EPO})^{52}$, avalado por estudios in vitro que muestran el efecto de citoquinas inflamatorias, como la IL-1, TNF- $\alpha$ e interferón $\gamma$ (IFN- $\gamma$ ) en la disminución de la diferenciación y proliferación de colonias progenitoras eritroides ${ }^{63}$. Por otro lado, una inadecuada capacidad de respuesta de la medula a la EPO, además de una disminución de la vida media de los eritrocitos maduros y alteración del metabolismo de $\mathrm{Fe}$ (deficiencia funcional de $\mathrm{Fe}$ ) con disminución de Fe disponible para eritropoyesis $^{53}$ son otros factores causales de la inhibición de la eritropoyesis.

Una sobreproducción moderada de Hpc en ratones transgénicos o en ratones productores de Hpc por tumores, replica muchas de las características patogénicas de la AEC, incluida una anemia leve, hipocrómica, con retención de Fe en el bazo y respuesta de la médula ósea frente al estímulo de $\mathrm{EPO}^{64}$. Por lo tanto, in vitro e in vivo se ha demostrado que la Hpc está implicada en la mayoría de los efectos y cambios relacionados con Fe que son características patognomónicas de la AEC. 


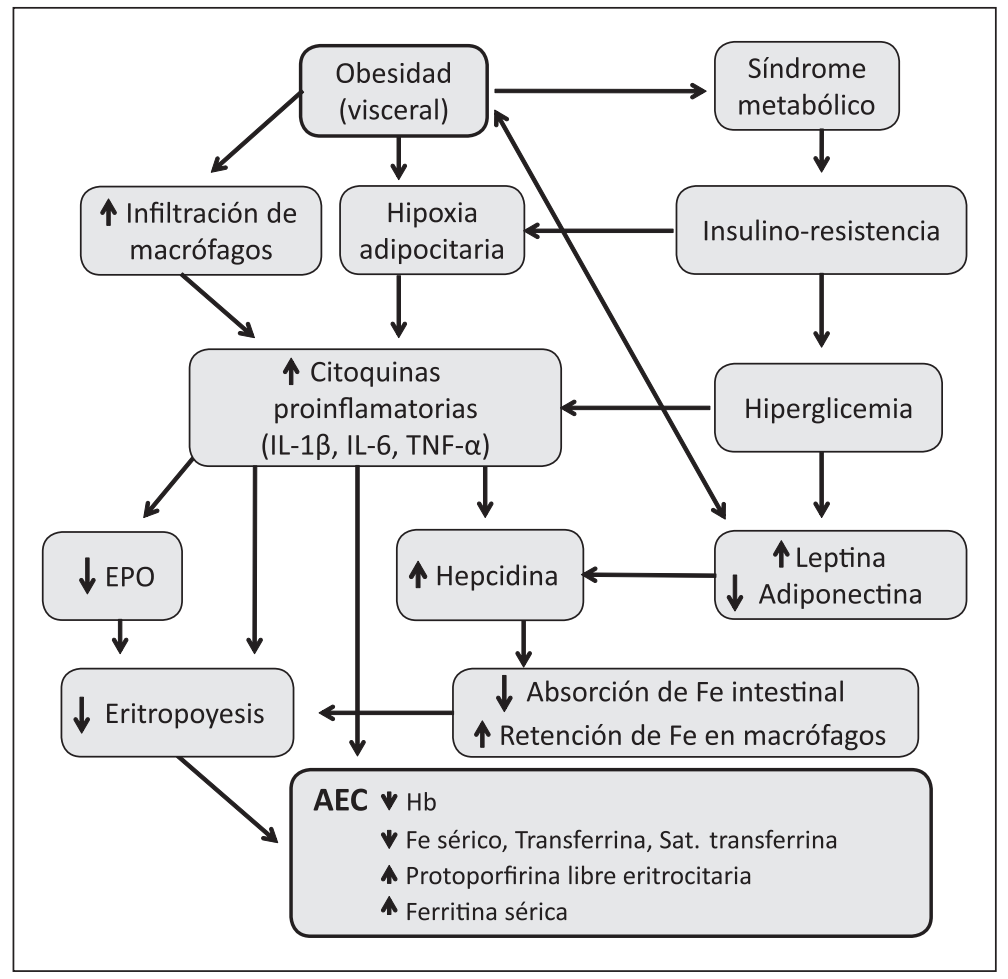

Figura 1. Patogenia de la AEC asociada a obesidad y sus comorbilidades como fenómeno multifactorial en el que intervienen diversos mecanismos donde la inflamación crónica modula el metabolismo férrico a través de la expresión de Hpc. EPO: eritropoyetina. AEC: anemia de las enfermedades crónicas.

\section{Perspectivas terapéuticas}

Las perspectivas terapéuticas aparecen como muy importantes y constituyen una etapa determinante en el campo de las enfermedades de la homeostasis del Fe, por lo que el desarrollo de terapias que permitan el manejo del sistema HpcFpn puede tener futuras implicancias.

En la actualidad se conoce una serie de estrategias para el tratamiento de la AEC en base a diferentes alternativas;

1) Inhibidores de la síntesis de $\mathrm{Hpc}$ a través del uso de 1a) ARN de interferencia y de oligonucleótidos antisentido como silenciadores del gen de Hpc y de la1b) neutralización de citoquinas (BMP o IL-6) y sus vías de señalización intracelular BMP6-HJV-SMAD e IL-6-STAT3 respectivamente, que estimulan su expresión.

2) Neutralizadores de la Hpc (antagonistas directos $\mathrm{Hpc}$ ) a través de $2 \mathrm{a}$ ) anticuerpos (Ac) Anti-Hpc, que se encuentran actualmente en Fase 1 de pruebas clínicas para el tratamiento de la AEC y 2 b) proteínas de secreción y oligonucleótidos capaces de unir y bloquear la Hpc.
3) Estimuladores de la eritropoyesis (EPO y agentes estimuladores de la eritropoyesis) que han demostrado suprimir la producción de Hpc en humanos, dentro de este grupo la vitamina $\mathrm{D}$ podría tener un efecto beneficioso en el incremento de la actividad eritropoyética y disminución de la inflamación.

4) Bloqueadores del sitio de unión a Fpn o inhibidores de la vía de internalización de Fpn (agonistas o estabilizadores de Fpn) que se plantean como estrategias emergentes para promover la actividad de Fpn, permitiendo el flujo continuo de Fe, sin embargo, la seguridad y eficacia de estos enfoques en humanos aún no han sido determinados ${ }^{62,65}$.

En consecuencia, desordenes en las vías de regulación de la expresión de Hpc determinan el metabolismo de Fe, por lo tanto, el estudio de sus mecanismos de acción se traduce en un mejoramiento del tratamiento de patologías asociadas $^{63}$. Finalmente, la patogenia de la AEC asociada a obesidad y sus comorbilidades es un fenómeno multifactorial en el que intervienen 
diversos mecanismos, donde la inflamación crónica modularía el metabolismo del Fe a través de la expresión de Hpc.

\section{Referencias}

1. Roy C. Anemia of Inflammation. Hematology Am Soc Hematol Educ Program 2010; 2010: 276-80.

2. Zafon C, Lecube A, Simó R. Iron in obesity. An ancient micronutrient for a modern disease. Obes Rev 2010; 11: 322-8.

3. Sanad M, Osman M, Gharib A. Obesity modulate serum hepcidin and treatment outcome of iron deficiency anemia in children: A case control study. Ital J Pediatr 2011; 37: 34 .

4. Wenzel BJ, Stults HB, Mayer J. Hypoferraemia in obese adolescents. Lancet 1962; 2: 327-8.

5. Menzie CM, Yanoff LB, Denkinger BI, Mchugh T, Sebring NG, Calis KA, et al. Obesity related hypoferremia is not explained by differences in reported intake of heme and nonheme iron or intake of dietary factors that can affect iron absorption. J Am Diet Assoc 2008; 108: 145-8.

6. Ganz T. Hepcidin, a key regulator of iron metabolism and mediator of anemia of inflammation. Blood 2003; 102: 783-8.

7. Nicolas G, Bennoun, M, Devaux I, Beaumont C, Grandchamp B, Kahn A, et al. Lack of hepcidin gene expression and severe tissue iron overload in upstream stimulatory factor 2 (USF2) knockout mice. Proc Natl Acad Sci 2001; 98: 8780-5.

8. Park $\mathrm{CH}$, Valore EV, Waring AJ, Ganz T. Hepcidin, a urinary antimicrobial peptide synthesized in the liver. J Biol Chem 2011; 276: 7806-10.

9. Krause A, Neitz S, Magert HJ, Schulz A, Forssmann WG, Schulz-Knappe P, et al. LEAP-1, a novel highly disulfidebonded human peptide, exhibits antimicrobial activity. FEBS 2000; 480: 147-50.

10. Ganz T, Nemeth E. Hepcidin and Disorders of Iron Metabolism. Annu Rev Med 2011; 62: 347-60.

11. Kemna E, Tjalsma H, Laarakkers C, Nemeth E, Willems $\mathrm{H}$, Swinkels D. Novel urine hepcidin assay by mass spectrometry. Blood 2005; 106: 3268-70.

12. Bekri S, Gual P, Anty R, Luciani N, Dahman M, Ramesh $\mathrm{B}$, et al. Increased adipose tissue expression of hepcidin in severe obesity is independent from diabetes and NASH. Gastroenterology 2006; 131: 788-96.

13. Robson KJ. Hepcidin and its role in iron absorption. Gut 2004; 53: 617-9.

14. Valore E, Ganz T. Posttranslational processing of hep- cidin in human hepatocytes is mediated by the prohormone convertase furin. Blood Cells Mol Dis 2008; 40: 132-8.

15. Pigeon C, Ilyin G, Courselaud B, Leroyer P, Turlin B, Brissot $\mathrm{P}$, et al. A new mouse liver-specific gene, encoding a protein homologous to human antimicrobial peptide hepcidin, is overexpressed during iron overload. J Biol Chem 2001; 276: 7811-9.

16. Nicolas G, Bennoun M, Porteu A, Mativet S, Beaumont C, Grandchamp B, et al. Severe iron deficiency anemia in transgenic mice expressing liver hepcidina. Proc Natl Acad Sci USA 2002; 99: 4596-601.

17. Wallace DF, Summerville L, Crampton EM, Frazer DM, Anderson GJ, Subramaniam VN. Combined deletion of Hfe and transferrin receptor 2 in mice leads to marked dysregulation of hepcidin and iron overload. Hepatology 2009; 50: 1992-2000.

18. Truksa J, Lee P, Beutler E. Two BMP responsive elements, STAT, and bZIP/HNF4/COUP motifs of the hepcidin promoter are critical for BMP, SMAD1, and HJV responsiveness. Blood 2009, 113: 688-95.

19. Babitt JL, Huang FW, Wrighting DM, Xia Y, Sidis Y, Samad TA, et al. Bone morphogenetic protein signaling by hemojuvelin regulates hepcidin expression. Nat Genet 2006; 38: 531-9.

20. Nemeth E, Valore EV, Territo M, Schiller G, Lichtenstein A, Ganz T. Hepcidin, a putative mediator of anemia of inflammation, is a type II acute phase protein. Blood 2003; 101: 2461-3.

21. Stoian I, Manolescu B, Atanasiu V, Lupescu O, Buşu C Rom J. IL-6-STAT-3-hepcidin: linking inflammation to the iron metabolism. Intern Med 2007; 45: 305-9.

22. Verga Falzacappa MV, Vujic Spasic M, Kessler R, Stolte J, Hentze MW, Muckenthaler MU. STAT3 mediates hepatic hepcidin expression and its inflammatory stimulation. Blood 2007; 109: 353.

23. Donovan A, Lima CA, Pinkus JL, Pinkus GS, Zon LI, Robine $\mathrm{S}$, et al. The iron exporter ferroportin/Slc40a1 is essential for iron homeostasis. Cell Metabolism 2005; 1 : 191-200.

24. Nemeth, E. Targeting the hepcidin-ferroportin axis in the diagnosis and treatment of anemias. Adv Hematol 2010; 2010: 750643

25. De Domenico I, Zhang TY, Koening CL, Branch RW, London N, Lo E, et al. Hepcidin mediates transcriptional changes that modulate acute cytokine-induced inflammatory responses in mice. J Clin Invest 2010; 120: 2395-405.

26. Ganz T. Molecular pathogenesis of anemia of chronic disease. Pediatr Blood Cancer 2006; 46: 554-7.

27. Laftah AH, Ramesh B, Simpson RJ, Solanky N, Bahram 
S, Schümann K, et al. Effect of hepcidin on intestinal iron absorption in mice. Blood 2004; 103: 3940-4.

28. Pinhas-Hamiel O, Newfield RS, Koren I, Agmon A, Lilos P, Phillip M. Greater prevalence of iron deficiency in overweight and obese children and adolescents. Int J Obes Relat Metab Disord 2003; 27: 416-8.

29. Ausk KJ, Ioannou GN. Is obesity associated with anemia of chronic disease? A population-based study. Obesity 2008; 16: 2356-61.

30. Lecube A, Carrera A, Losada E, Hernández C, Simo R, Mesa J. Iron deficiency in obese postmenopausal women. Obesity 2006; 14: 1724-30.

31. Dandona P, Aljada A, Bandyopadhyay A. Inflammation: the link between insulin resistance, obesity and diabetes, Trends Immunol 2004; 25: 4-7.

32. Weisberg SP, McCann D, Desai M, Rosenbaum M, Leibel RL, Ferrante AW Jr. Obesity is associated with macrophage accumulation in adipose tissue. J Clin Invest 2003; 112: 1796-808.

33. Trayhurn P. Endocrine and signalling role of adipose tissue: new perspectives on fat. Acta Physiol Scand 2005; 184: 285-93.

34. Hintze KJ, Snow D, Nabor D, Timbimboo H. Adipocyte hypoxia increases hepatocyte hepcidin expression. Biol Trace Elem Res 2010; 143: 764-71.

35. Luciani N, Brasse-Lagnel C, Poli M, Anty R, Lesueur C, Cormont M, et al. Hemojuvelin: a new link between obesity and iron homeostasis. Obesity 2011; 19: 1545-51.

36. Gabay C, Kushner I. Acute-phase proteins and other systemic responses to inflammation. N Engl J Med 1999; 340: 448-54.

37. Yudkin JS, Stehouwer CD, Emeis JJ, Coppack SW. Creactive protein in healthy subjects: associations with obesity, insulin resistance, and endothelial dysfunction: a potential role for cytokines originating from adipose tissue? Arterioscler Thromb Vasc Biol 1999; 19: 972-8.

38. Zandman-Goddard G, Shoenfeld Y. Ferritin in autoinmune diseases. Autoimmun Rev 2007; 6: 457-63.

39. Iwasaki T, Nakajima A, Yoneda M, Yamada Y, Mukasa K, Fujita K, et al. Serum ferritin is associated with visceral fat area and subcutaneous fat area. Diabetes Care 2005; 28: 2486-91.

40. de Luca C, Olefsky JM. Inflammation and insulin resistance. FEBS Lett 2008; 582: 97-105.

41. Davis RJ, Corvera S, Czech MP. Insulin stimulates cellular iron uptake and causes the redistribution of intracellular transferrin receptors to the plasma membrane. J Biol Chem 1986; 261: 8708-11.

42. Wilson JG, Lindquist JH, Grambow SC, Crook ED, Maher JF. Potential role of increased iron stores in diabetes. Am J Med Sci 2003; 325: 332-9.
43. McCarty MF. Hyperinsulinemia may boost both hematocrit and iron absorption by up-regulating activity of hypoxia-inducible factor- $1 \alpha$. Med Hypotheses $2003 ; 61$ : 567-73.

44. Alberti KG, Zimmet P, Shaw J. IDF Epidemiology Task Force Consensus Group The metabolic syndrome-a new worldwide definition. Lancet 2005; 366: 1059-62.

45. Jehn M, Clark JM, Guallar E. Serum ferritin and risk of the metabolic syndrome in U.S. adults. Diabetes Care 2004; 27: 2422-8.

46. Ahima RS, Prabakaran D, Flier JS. Leptin. Annu Rev Physiol 2000; 62: 413-37.

47. Baumann H, Morella KK, White DW, Dembski M, Bailon PS, Kim H, et al. The full-length leptin receptor has signaling capabilities of interleukin 6-type cytokine receptors. Proc Natl Acad Sci USA 1996; 93: 8374-8.

48. Chung B, Matak P, McKie AT, Sharp P. Leptin increases the expression of the iron regulatory hormone hepcidin in HuH7 human hepatoma cells. J Nutr 2007; 137: 236670.

49. del Giudice EM, Santoro N, Amato A, Brienza C, Calabrò $\mathrm{P}$, Wiegerinck ET, et al. Hepcidin in obese children as a potential mediator of the association between obesity and iron deficiency. J Clin Endocrinol Metab 2009; 94: 5102-7.

50. Berg AH, Combs TP, Du X, Brownlee M, Scherer PE. ACRP30/adiponectin; adipokine regulating glucose and lipid metabolism. Trends Endocrinol Metab 2002; 13: 84-9.

51. Ye J, Gao Z, Yin J, He Q. Hypoxia is a potential risk factor for chronic inflammation and adiponectin reduction in adipose tissue of ob/ob and dietary obese mice. Am J Physiol Lung Cell Mol Physiol 2007; 293: E1118-E1128.

52. Weiss G, Goodnough LT. Anemia of chronic disease. N Engl J Med 2005; 352: 1011-23.

53. Price EA, Schrier SL. Unexplained aspects of anemia of inflammation. Adv Hematol 2010; 2010: 508739.

54. Skikne BS. Serum transferrin receptor. Am J Hematol 2008; 83: 872-5.

55. Theurl I, Aigner E, Theurl M, Nairz M, Seifert M, Schroll A, et al. Regulation of iron homeostasis in anemia of chronic disease and iron deficiency anemia: diagnostic and therapeutic implications. Blood 2009; 113: 5277-8.

56. Cullis JO. Diagnosis and management of anaemia of chronic disease: current status. Br J Haematol 2011; 154: 289-300.

57. Cook JD. Diagnosis and management of iron-deficiency anaemia. Best Pract Res Clin Haematol 2008; 18: 319-32.

58. Zimmermann MB. Methods to assess iron and iodine status. Br J Nutr 2008; 99: S2-S9. 
59. Verga Falzacappa MV, Casanovas G, Hentze MW, Muckenthaler MU. A bone morphogenetic protein (BMP)responsive element in the hepcidin promoter controls HFE2-mediated hepatic hepcidin expression and its response to IL-6 in cultured cells. J Mol Med 2008; 86: 531-40.

60. Collins JF, Wessling-Resnick M, Knutson MD. Hepcidin regulation of iron transport. J Nutr 2008; 138: 2284-8.

61. Hugman A. Hepcidin: an important new regulator of iron homeostasis. Clin Lab Haematol 2006; 28: 75-83.

62. Ganz T, Nemeth E. The hepcidin-ferroportin system as a therapeutic target in anemias and iron overload disorders. Hematology Am Soc Hematol Educ Program 2011; 2011: 538-42.

63. Weiss, G. Iron metabolism in the anemia of chronic disease. Biochim Biophys Acta 2009; 1790: 682-93.

64. Roy CN, Mak HH, Akpan I, Losyev G, Zurakowski D, Andrews NC. Hepcidin antimicrobial peptide transgenic mice exhibit features of the anemia of inflammation Blood 2007; 109: 4038-44.

65. Sun CC, Vaja V, Babitt JL, Lin HY. Targeting the hepcidin-ferroportin axis to develop new treatment strategies for anemia of chronic disease and anemia of inflammation. Am J Hematol 2012; 87: 392-400. 